\title{
Combined Spectral Resonances of Signaling Proteins' Amino Acids in the ERK-MAP Pathway Reflect Unique Patterns That Predict Peak Photon Emissions and Universal Energies
}

\author{
Michael A. Persinger ${ }^{\star}$, Nirosha J. Murugan, Lukasz M. Karbowski \\ Laurentian University, Sudbury, Ontario P3E 2C6, Canada \\ *E-mail address: mpersinger@laurentian.ca
}

\begin{abstract}
The duality of matter-energy as particle-waves was applied to the classic ERK-MAP signaling pathways between the plasma cell membrane and the nucleus and was tested with Cosic's Resonance Recognition Method. Spectral analyses of sequences of pseudopotentials that reflect de-localized electrons of amino acids for the 11 proteins in the pathway were computed. The spectral power density of the terminal protein (cFOS) was shown to be the average of the profiles of the precursor proteins. The results demonstrated that in addition to minute successive alterations in molecular structure wavefunctions and resonant patterns can also describe complex molecular signaling pathways in cells. Different pathways may be defined by a single resonance profile. The separations between the peaks of wavelengths from Cosic's predictions for photon emissions in the visible spectrum that define the ERK-MAP pathway were within the range of $10^{-20} \mathrm{~J}$. This quantity has been shown to be a fundamental unit of energy within the universe. The involvement of photon patterns indicates that non-local effects could accompany the serial causality (locality) assumed to connect molecular pathways.
\end{abstract}

Keywords: Cosic Resonance Recognition Mechanism; ERK-MAP signaling pathway; $10^{-20} \mathrm{~J}$; wavefunctions; photon emissions; particle-wave phenomena

\section{INTRODUCTION}

The substance or subject matter of Science is based upon enumeration. The patterns of these numbers in space-time determine the concepts that define phenomena. The simplified constructs for understanding the immense variations of those measurements constitute the models of perception and understanding. Scientists have usually assumed that the greater the congruence between predictions from a model and the characteristics of the phenomenon being measured, the more accurate and valid are the presumptions of the model.

For example, to explain the retrograde motion of the planet Mars from the reference of the earth as the center of the universe Ptolemy was required to add epicycles or additional 
subcircles upon the geocentric orbital circles. Copernicus' heliocentric system accommodated the retrograde phenomena by recognizing the different distances of the earth's and Mars' orbits with respect to their relative positions within their orbits. Although both systems predicted the phenomenological and observational aspects of Mars' retrograde motion, the system of Copernicus was ultimately demonstrated to be more consistent with the larger body of measurements.

The two major components for human reasoning, perception, and description of reality have been space and time. Discrete increments of space are allocated to matter or particles.

The spatial patterns of these units of matter or particles determine their function. Discrete increments of time are allocated to complex waves and fields. The temporal patterns of these fields and waves determine their function. The combinations of these qualitatively discrete categories into a blending field, such as defined by Minkowski's four-dimensional manifold of space-time representation that became instrumental to relativity theories, accommodate the simultaneous dualism of de Broglie matter waves whereby a unit particle, such as an electron, could be either a particle or a wave.

The contemporary approach for molecular biology is serial causality in order to accommodate the demands of locality. Consequently for information or a change in stimulus characteristics to move from one boundary, such as the plasma cell membrane, to an internal boundary, such as the nuclear membrane, a series of spatial interfaces must occur. These interfaces which usually involve the addition or removal of an atomic or molecular component, such as a phosphate group or a proton, occur through a succession of different proteins. These signaling pathways, or more accurately "networks", are aggregates of molecular sequences that could be defined as fields in four-dimensional space.

Starting with some component (A) within the plasma cell membrane, A affects B and B affects C....N until the terminus (the nucleus) is reached. The "information" contained within that series affects the dynamics of the nucleus to initiate transcription and consequently to control the entire cell. The whole of the different proteins within this succession are often described as signaling pathways. For many of the signaling pathways that have been preserved in life forms for the last few billion years the numbers of proteins range from about 8 to 15 .

Traditionally molecules with similar spatial patterns (structure) are assumed to exhibit similar functions and molecules of markedly dissimilar structures share minimal functions. However Irena Cosic [1] observed marked discrepancies for this central assumption. She addressed this discrepancy by assigning each amino acid with a calculated psuedopotential value based upon the characteristics of de-localized electrons. When these sequence values were spectral analyzed through Fast Fourier Transforms the resulting spectral power densities (SPDs) predicted the wavelength and hence the frequency of the potential photons that can be measured to be emitted from that molecule.

Subsequent experiments by Dotta et al [2] showed that during the habituation to ambient temperatures melanoma cells that had been removed from standard incubation conditions $\left(37^{\circ} \mathrm{C}\right)$ shifted power densities across the visible spectrum. Protein enhancers or suppressors for components of the cell whose peak wavelength had been predicted by Cosic's model of Resonance Recognition for Molecules (RRM) increased or decreased the photon radiant flux densities from those cells within the range of accuracy discern by the filters employed in the measurements.

In a manner similar to the difference between Ptolemy's and Copernicus' explanations for the retrograde motions of Mars, we predicted that there may be two models for the accurate prediction of the intercalation between components of signaling pathways in living 
cells. Both involve transmission of energy. The molecular approach presumes this transmission is completed by discrete addition or removal of matter (a molecule or proton/electron). The Cosic approach assumed that the energy is distributed through the resonance field created by the spatial pattern of the amino acids that constitute the proteins. The accompanying oscillating field could be electromagnetic in nature.

One process would involve the sequential exchange of a quantity of energy from molecule to molecule. The other would involve the summation and averaging of the spectral power density of the spatial order of the amino acids that constitute these proteins. Like the de Broglie matter-waves both manifestations could exist. Whereas the former would require locality to be effective, the latter, if photons were directly involved, could allow the introduction of non-local processes or "entanglement" [3] within the cell between the outer (plasma cell membrane) and inner (nuclear membrane) boundaries. Here we present quantitative evidence for this possibility.

\section{TRANSFORMATON OF THE MAP-ERK PATHWAY TO COSIC'S SERIAL PSEDUOPOTENTIALS}

The signaling pathway presently designated as MAPK (Mitogen-activated protein kinases), originally labeled as ERK (extracellular signal-regulated kinases) is a "chain" of proteins that mediates changes or "information" from a receptor on the cell's surface to the DNA within the nucleus of the cell. In general the sequence of proteins (mass in kDaltons in parentheses) from the surface of the cell to the nucleus are: VEGF $(234 \mathrm{kD})$, TRK $(348 \mathrm{kD})$, HRas (191 kD), CRAF (640 kD), MEK1 (395 kD), MEK2 (402 kD), ERK1 (362 kD), ERK2 (343 kD), CREB (343 kD), cFOS (383 kD) and PLA2 (808 kD). The meaning of each acronym can be found elsewhere [4].

The latter two proteins, cFOS and PLA2 (phospholipase A2) are considered to act in different spaces but to be determined by the components of the pathway. CFOS affects the nucleus while PLA2 involves more cytoplasmic activity. The molecular component that is shared in the serial sequence from a matter-molecular perspective is the addition of a phosphate group to the neighboring protein that could be considered an aggregate equivalent of a Grotthuss-like chain.

To discern the spatial spectral power density of the amino acid sequence of each of these components of the pathway, each amino acid for each molecule was assigned the psuedopotential value as described by Cosic $[1,5]$. The pseudo potential is the estimated electron-ion interaction potential (EIIP) that describes the average energy states of all valence electrons for each amino acid. The formula has been published [1]. What may be important from an astronomical perspective, particularly if Ernst Mach's ideas [6] are considered, is contribution of the change of momentum of the delocalized electrons in the interaction.

Because spectral analyses with the algorithm we employed (SPSS-16 PC) required equal case numbers and the different proteins exhibit different lengths of amino acids, all proteins whose lengths were less than the longest one in the pathway were extended by sequential adding of the values (a type of "statistical PCR, or polypeptide chain reaction) so that all sequences were equal length. Spectral analyses were then completed. The real spatial "frequency" was obtained by dividing $0.38 \mathrm{~nm}$ by the spectral frequency unit produced by the software. The value of $0.38 \mathrm{~nm}$ was considered to be the average width of an amino acid.

For theoretical aesthetics we assumed that a feasible distance for de-localized electrons involving an average bond length (L) of $\sim 0.25 \mathrm{~nm}$ would be $\pi \mathrm{L}(\sim 0.8 \mathrm{~nm})$. The SPD for each 
of the 11 proteins were plotted as a function of the real spatial frequency for the range $<0.8$ $\mathrm{nm}$. This interval contained 50 successive spectral power densities each separated by 0.001 base frequency units or $\Delta f$. To test the concept of shared endpoints we employed canonical correlation. The last two components of the MAPK-ERK pathway, cFOS and PLA2 were designated as the dependents and the remaining variables were considered the predictors or covariates.

Table 1. Loading (correlation) coefficients for each protein within the ERK-MAP pathway upon the primary root extracted by canonical correlation for the two terminal components (cFOS and PLA2) of the pathway.

\begin{tabular}{|c|c|}
\hline Dependent & Precursors (Independent) \\
\hline \multirow{4}{*}{ cFos +0.65} & VEGF 0.41 \\
\cline { 2 - 2 } & TRK 0.43 \\
\cline { 2 - 2 } & H-Ras 0.07 \\
\cline { 2 - 2 } & C-Raf -0.02 \\
\hline \multirow{4}{*}{ Pla2 -0.52 } & MEK1 -0.14 \\
\cline { 2 - 2 } & MEK2 0.40 \\
\cline { 2 - 2 } & ERK1 0.43 \\
\cline { 2 - 2 } & ERK2 0.60 \\
\hline \multirow{4}{*}{} & CREB -0.26 \\
\hline
\end{tabular}

The results are shown in Table 1 . The only statistically significant $(\mathrm{p}<.01)$ root extracted for the canonical correlation indicated that the spatial spectral density of cFOS, the one protein associated with nuclear changes, and PLA2, the protein associated with cytoplasmic activity, were negatively correlated ("loaded") on the root. There is additional evidence from classical biomolecular interpretations that the activity of the two proteins were negatively correlated such that as one increases the other decreases.

However most biomolecular methodogies do not often differentiate time course and hence the reciprocal relationship, if it occurred within the millisecond range for example, would not be differentiated. To discern this dynamic the temporal increment $(\Delta t)$ of the measurement must be less than the intrinsic frequency of the fluctuation. With larger $\Delta$ ts the measurer would observe only an increase in both protein activities.

The resonance pattern for cFOS was significantly associated with that of VEGF (the first protein in the signaling pathway) as well as TRK, MEK2, ERK1, and ERK2. On the other hand the resonance pattern of PLA2 was negatively correlated with the resonance pattern of those proteins. The resonance patterns for H-Ras, C-Raf, MEK1 and CREB were not significantly correlated with the patterns of either cFOS or PLA2.

The correlogram or scattergram of the relationship between the spatial resonance or spectral densities of the dependent variables (cFos and PLA2) and the precursors of the root is shown in Figure 1. This was completed by multiplying the unstandardized discriminant function coefficient score for each variable for the dependent variables (and adding the constant) and by multiplying the specific coefficient for each of the independent variables to 
obtain that function. The Pearson correlation was $r=0.67(\mathrm{p}<.001)$. The data are represented as standardized scores.

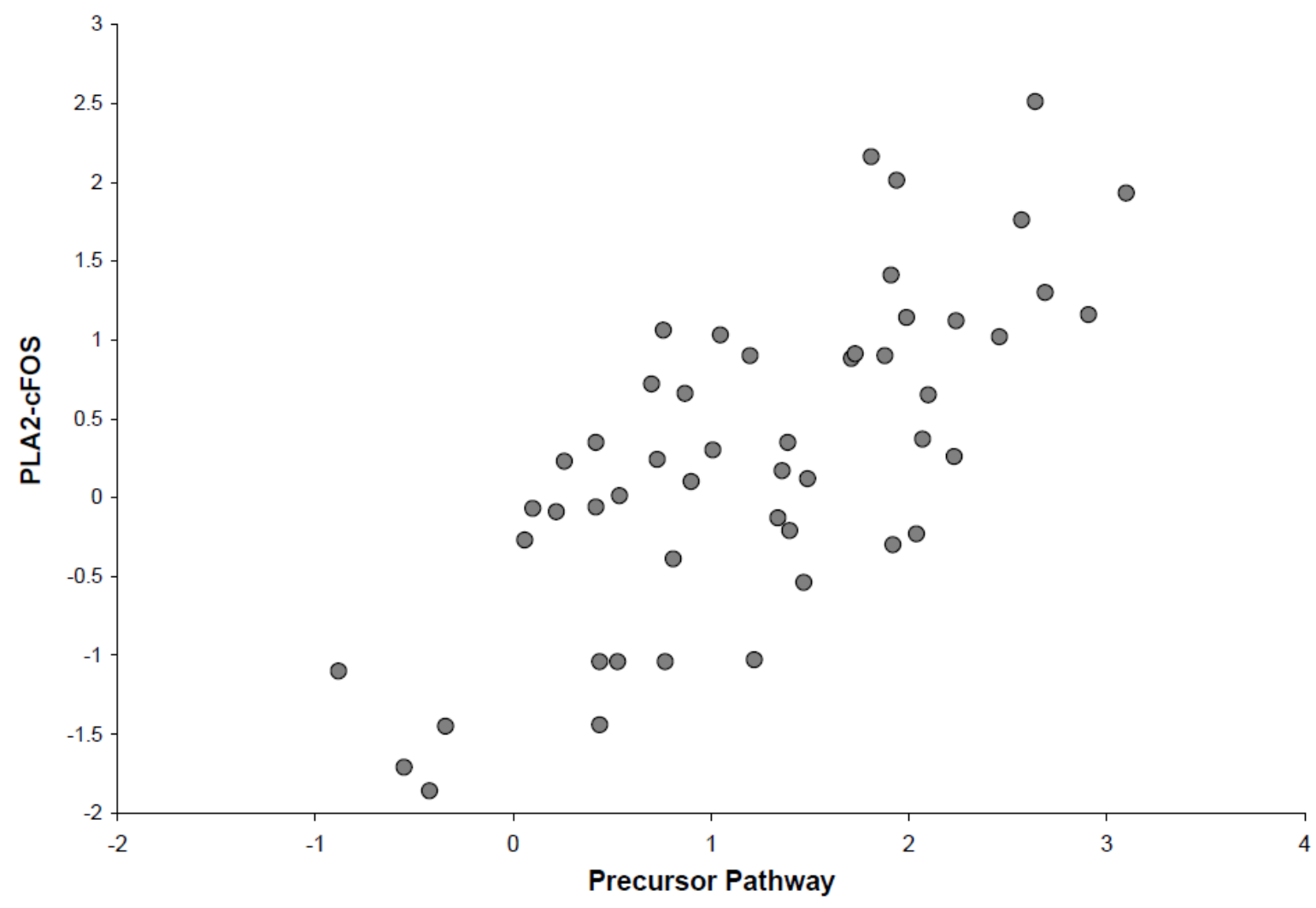

Figure 1. Scattergram of the correlation between the combination of the spectral power densities (SPDs) of two dependent variables (cFOS and PLA2) on the vertical axis and the SPDs for the precursor pathway proteins (horizontal axis) extracted in the first root.

\section{WEIGHTED LINEAR ADDITION OF SPECTRAL DENSITY PATTERNS (SPDS) OF PRECURSORS PREDICT SPDS OF TERMINAL PROTEIN}

Considering the negative correlation between the two proteins (cFOS, and PLA2) whose positions are often allocated at the end of the causal sequences between changes in membrane activity and induction of alterations in DNA function, multiple regression (step wise) analyses were completed for each independently. For cFOS multiple regression with cFOS as the dependent variable and the SPDs for each of the 9 precusors proteins as independent variables, resulted in a strong multiple $\mathrm{r}(\mathrm{r}=0.79)$ that was statistically significant $[\mathrm{F}(5,45)=15.18, \mathrm{p}<.001 ; 59 \%$ of variance explained $]$.

The congruence between the predicted SPDs for each spectral frequency unit $(\Delta f)$ for cFOS and the actual value for the cFOS molecule itself is shown in Figure 2. The equation including the partial regression coefficients (partial slopes) was 0.74(ERK2) -0.51(MEK1) 0.57 (ERK1) +0.24 (VEGF) +0.17 (Hras) +0.02 . To ensure the specificity of the congruence of the spectral increments $(\Delta f \mathrm{~s})$, because there may have been a mild phase shift for the different proteins, lag/lead analyses were completed. With the second lag as the dependent variable for cFOS, the SPDs for \pm 3 spectral units or $\Delta f$ s for each molecule were entered as predictor 
variables. There was no statistically significant increase in the accuracy of the prediction (multiple $\mathrm{r}=0.83$ ).

When the SPDs for PLA2 was employed as the dependent variable and the SPDs of the same nine variables were entered by the stepwise procedure only one variable entered: ERK2 $[\mathrm{F}(1,49)=10.69, \mathrm{P}<.01 ; 18 \%$ of the variance explained $]$. When cFOS was added to list of predictor variables, the multiple $\mathrm{R}[\mathrm{F}(2,48)=12.01, \mathrm{p}<.001]$ value increased to $\mathrm{MR}=0.58$ (31\% of the variance explained). The equation was $-.58(\mathrm{cFOS})-0.24$ (ERK2) +0.06 and indicated that in terms of shared numerical variance the resonance characteristics of PLA2 were affected by that of cFOS.

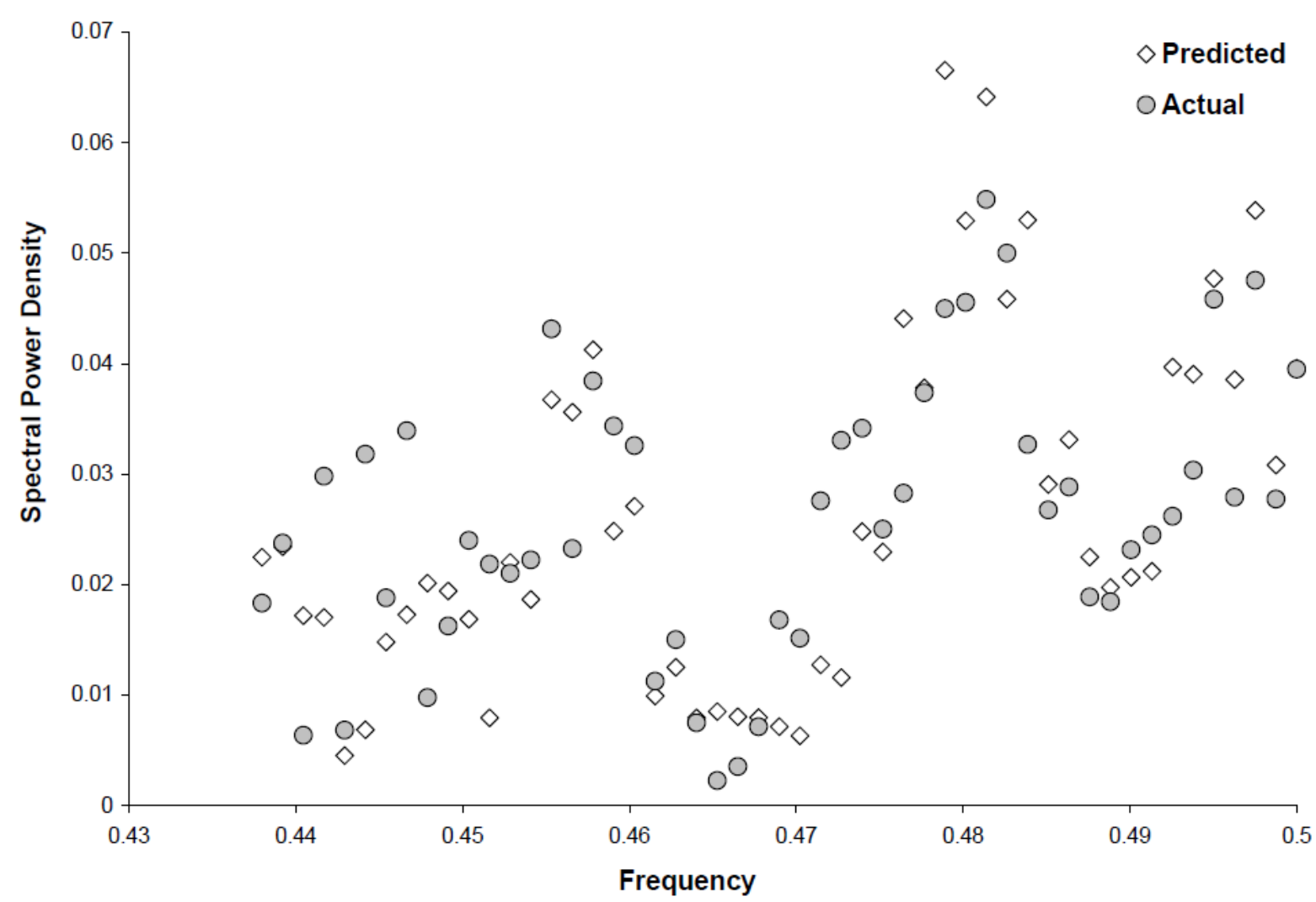

Figure 2. Spectral Power Densities (SPDs) as a function of numerical frequency for the actual cFOS protein molecule (closed circles) and the predicted SPDs (open diamonds) based upon weighted linear combinations of the SPDs of antecedent proteins in the pathway.

Even when $\pm 3 \mathrm{lag} / \mathrm{leads}$ for the $\Delta f \mathrm{~s}$ for the SPDs for each protein (except $\mathrm{cFos}$ ) were added as potential predictor variables the multiple $\mathrm{r}(0.57)$ did not change significantly. However when cFOS and its \pm 3 lags were added, the predicted multiple $r$ increased to 0.88 $[\mathrm{F}(6,39)=23.14, \mathrm{p}<.001 ; 75 \%$ of variance explained $]$. The equation was cFOS, lead 1 unit ($0.63)$, VEGF (0.61), cFOS, lagged 2 units (-1.12), CREB, lagged 3 units (-0.50), Hras (0.45), ERK2 $(-0.24)+0.08$ (constant). The results are shown in Figure 3. 


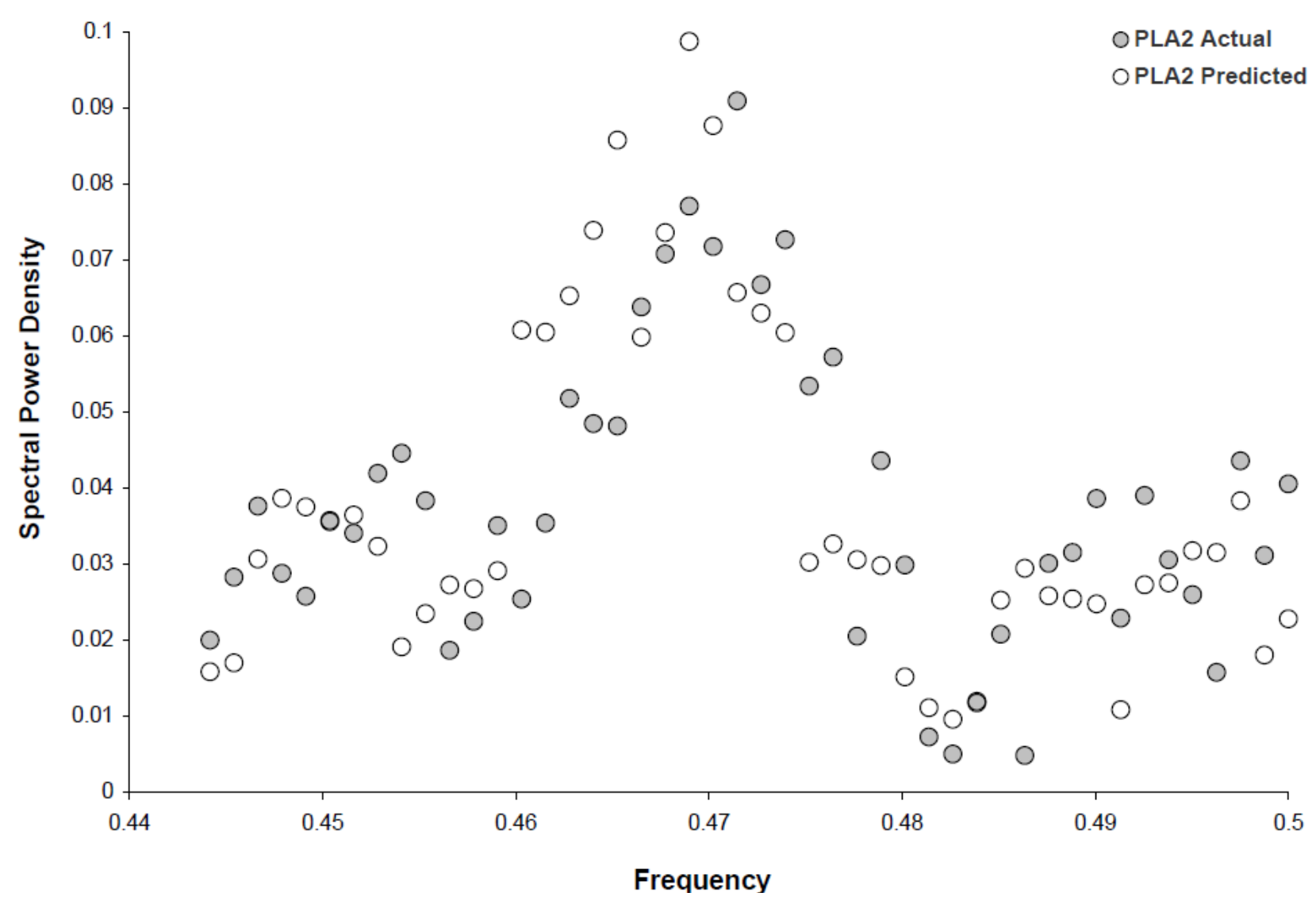

Figure 3. The correlogram of the predicted Spectral Power Density values (open circles) for Phospholipase (PLA2) protein and the actual SPD values (closed circles) for that protein.

To verify that the spectral composition of the average of the SPDs for cFOS and PLA2 were indeed independent with respect to their constituent (precursor) proteins in the pathway, the mean of the SPDs for cFOS and PLA2 were entered as dependent variables. No equation was generated with a pin (probability to enter) level of $p<0.05$ from the eight precursor variables. In effect this aggregate average, although its components were significantly associated with the SPDs for the precursor molecules in the pathway, were not revealing. The orthogonal ("antiparallel") association between these two terminal proteins in combination functionally cancelled or "occluded" the conspicuous relationship with the precursors.

There are four major indications from these results. First, the relationship between the proteins within a classic "signaling pathway" that leads to cFOS effects on the nucleus produce a combined resonance pattern that overlaps with the specific resonance pattern of the cFOS molecule. This suggests that molecular pathways can display wave-like properties where components can be decomposed and re-composed into an aggregate that reflects the whole. In other words, the whole is a composite of the weighted mean of the parts.

The second conclusion is that the spectral increments are relatively precise for the central component of the pathway. Adding the lag or lead values (each equivalent to a hypothetical change of $\Delta f=0.001$ for the spectral increments of the precursor molecules did not significantly change their final correlation (similar resonance pattern) with the cFOS molecule. Hence the likelihood that "random" variables entered the equation could be considered minimal. 
Third the PLA2 component which has often been paired according to traditional biomolecular interpretations with cFOS is not independent of cFOS. Its SPD is required to accommodate PLA2's resonance. In addition, the components from the precursor molecules may be phase shifted by about $0.003 \mathrm{~nm}$ of an equivalent wavelength (in $\mathrm{nm}$ ) or one or two SPD frequency units $(0.001)$. This would be equivalent to $3 \cdot 10^{-12} \mathrm{~nm}$ which approaches the Compton $\lambda\left(2.42 \cdot 10^{-12} \mathrm{~m}\right)$ for an electron.

These results indicate that the SPD for the Rydberg-derived pseudopotentials of amino acids in the traditional terminal proteins, cFOS and PLA2 displayed distinct resonance patterns. As shown in Figure 4, the SPDs profiles for the two proteins are negatively correlated $(r=-0.60)$, or, from a wave perspective, almost maximally out of phase.

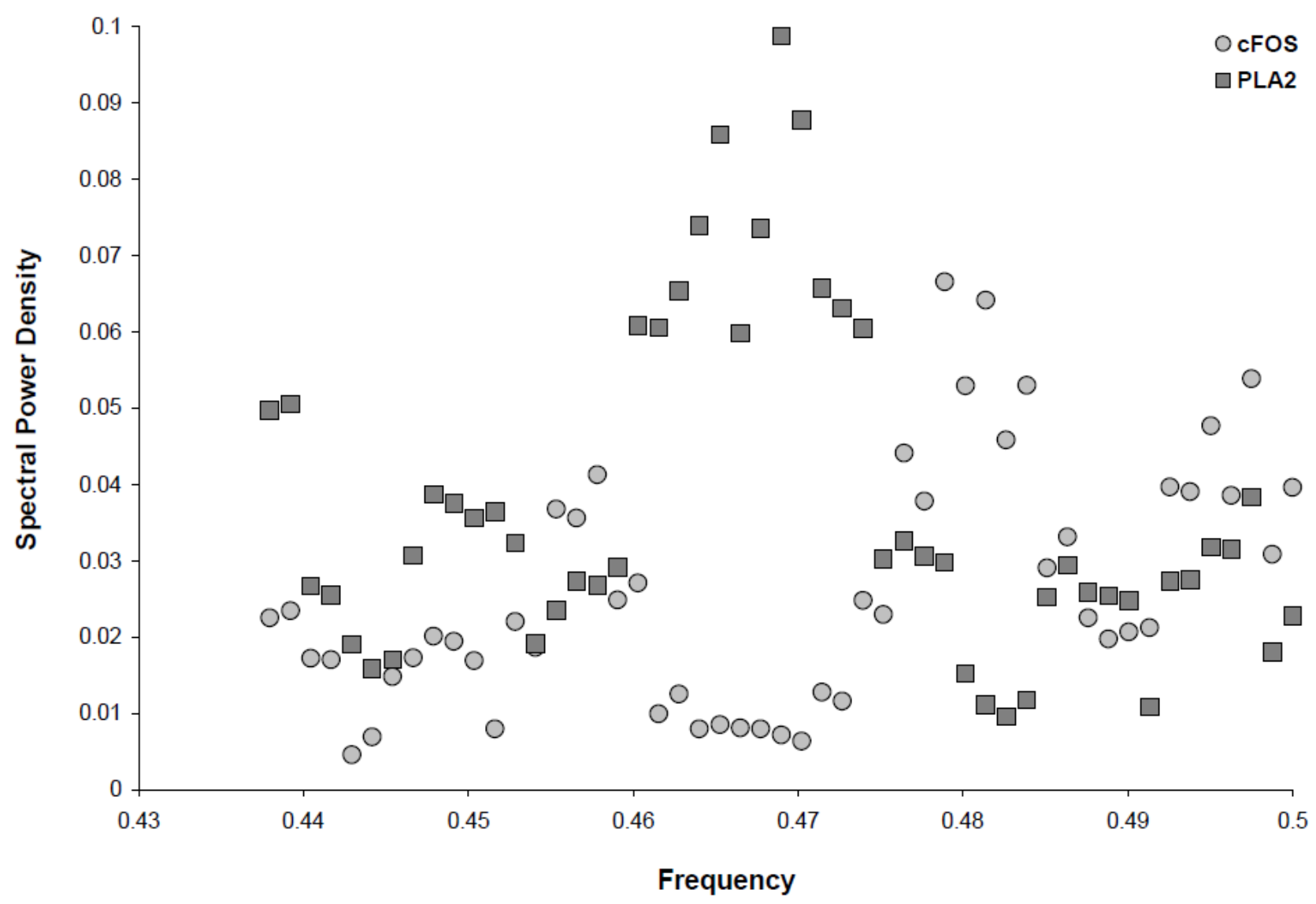

Figure 4. Overlap of the Spectral Power Densities for the cFOS (light circles) and PLA2 (dark squares) molecules according to Cosic's method as a function of base frequency for distances of $<0.8$ $\mathrm{nm}$ to accommodate de-localized electrons.

\section{PREDICTIONS OF PEAK PHOTON EMISSION FROM RESONANCE PATTERNS OF SIGNALING PATHWAYS}

According to Cosic [1,5], each specific biological function within a protein (or DNA) is characterized by one frequency that in turn predicts a peak wavelength for photon emissions. From an aggregate or field perspective specific biological functions of a "serial" pathway might be described by a specific spectral profile or pattern of peak frequencies. According to 
Cosic, the peak wavelength $\lambda$ for photon emission, which we have demonstrated to be valid through direct experimental manipulation is:

$$
\lambda=K \cdot f_{\text {rrm }}(1)
$$

where $\mathrm{K}$ is the constant 201 and $\mathrm{f}_{\text {rrm }}$ is the numerical frequency obtained from the spectral analysis.

For the peak SPD for cFOS, which could be predicted by the weighted linear combination of the precursor proteins in the ERK-MAP pathway, the peak numerical frequencies were $.458(.455-.460), 0.481(0.476-0.484)$ and $0.498(.497-0.498)$. The central value $(0.481)$ was more than two standard deviations above the central tendency (mean) for the sequence. The other two peaks were more than one standard deviation above the mean.

According to the Cosic formula (1), the peak wavelength $(\lambda)$ for photons for the cFOS protein would be $438.9 \mathrm{~nm}, 417.9 \mathrm{~nm}$, and $403.6 \mathrm{~nm}$, respectively. By dividing these values into the velocity of light in a vacuum, frequencies are obtained. These frequencies multiplied by Planck's constant $\left(6.626 \cdot 10^{-34} \mathrm{~J} \cdot \mathrm{s}\right)$ resulted in energies that were $4.52,4.76$, and $4.92 \cdot 10^{-19}$ $\mathrm{J}$, respectively. From a theoretical perspective what is much more important is difference in energies between the power peaks. They would be $2.4 \cdot 10^{-20} \mathrm{~J}$ and $1.6 \cdot 10^{-20} \mathrm{~J}$ which is within the range of the second shell energies associated with the movement of protons through water [7] and is considered to be a fundamental energetic unit across hyperdimensional space [8].

On the other hand, the peak numerical frequency for PLA2 was 0.465 (range 0.460 to 0.474 ). There was a smaller peak around 0.438 . The associated photon wavelengths would be $432 \mathrm{~nm}$ and $458 \mathrm{~nm}$. The corresponding energies are $4.6 \cdot 10^{-19} \mathrm{~J}$ and $4.34 \cdot 10^{-19} \mathrm{~J}$. The difference between these two corresponding energies is $2.6 \cdot 10^{-20} \mathrm{~J}$.

\section{THE ASTROBIOLOGICAL SIGNIFICANCE OF $10^{-20}$ JOULE PHASE SHIFT INCREMENTS WITHIN RESONANCE PATTERNS}

The occurrence of specific peaks of predicted wavelengths within the ultraviolet boundary of the visible spectrum from the combined SPDs of the molecules that reflect the SPD profile of cFOS has local and non-local applications. Cosic had stated that the frequencies predicted by the RRM could represent oscillations of some physical field which propagates through water dipoles. This field could be electromagnetic in nature. Both theoretical and empirical [2] approaches support her contention.

This "electromagnetic nature" may be intrinsic to the physical chemistry of water and its interactions with solutes. The division of the magnetic moment of a proton $\left(1.41 \cdot 10^{-26}\right.$ $\left.\mathrm{A} \cdot \mathrm{m}^{2}\right)$ by the unit charge $\left(1.6 \cdot 10^{-19} \mathrm{~A} \cdot \mathrm{s}\right)$ results in a term of diffusion $\left(0.88 \cdot 10^{-7} \mathrm{~m}^{2} \cdot \mathrm{s}^{-1}\right)$. When applied to the average viscosity of water $\left(6.3 \cdot 10^{-4} \mathrm{~kg} \cdot \mathrm{m}^{-1} \cdot \mathrm{s}^{-1}\right)$ for biological temperatures the force would be $5.54 \cdot 10^{-11} \mathrm{~kg} \cdot \mathrm{m} \cdot \mathrm{s}^{-2}$. If this force were applied across the distance of two $\mathrm{O}-\mathrm{H}$ bonds $\left(1.92 \cdot 10^{-10} \mathrm{~m}\right)$ that would constitute the water molecule, the energy would be $\sim 1.1 \cdot 10^{-20}$ J. If that force was applied over the estimated width of an amino acid the energy would be $\sim 2 \cdot 10^{-20} \mathrm{~J}$ [9]. This value is within the range of the differences in energy between the peak photon emissions from the cFOS complex as predicted by the Resonance Recognition Model.

The possibility that fundamental quantities of energy that operate cellular mechanisms are the same as those found anywhere in the universe and hence relates the processes found within both astronomical and cellular phenomena has been primary ignored by modern perspectives. However this consistency would be consistent with Ernst Mach's concept of 
Prominence of the Universe or his principle that the behaviour of any component of the universe, presumably no matter how small, is determined by all of its parts. The concept is also consistent with the assumption that a physical field can exist anywhere.

We [10] have suggested that the description of the whole, in this case the consideration of the universe as a single spatial and temporal unit, simplifies the required geometries and mathematical descriptions, to basic equations. For example the mass of the universe $10^{52} \mathrm{~kg}$ multiplied by its width $10^{26} \mathrm{~m}$ and the square of the intrinsic Puthoff frequency $\left(10^{86} \mathrm{~s}^{-2}\right)$ results in a force of $10^{164} \mathrm{~N}$. The force per smallest unit (a Planck's voxel of $\sim 10^{-105} \mathrm{~m}^{-3}$ ) when all of these units within the volume of the universe $\left(10^{78} \mathrm{~m}^{3}\right)$ are considered, i.e., $10^{183}$ Planck's voxels in the universe, results in a force of $10^{-19} \mathrm{~N}$ per voxel. If this force is applied across the most fundamental wavelength of the universe, the $10^{-1} \mathrm{~m}$ displayed by neutral hydrogen, the energy is $\sim 10^{-20} \mathrm{~J}$. Inclusion of specific coefficients for thee above constituents does not change the order of magnitude for the solution.

The $10^{-20} \mathrm{~J}$ solution as a basic unit of energy transmission within the living physicalchemical system has been shown within several levels of discourse by Persinger [10]. For example the $\sim 10^{-12} \mathrm{~N}$ of electric force between two potassium ions whose single layer of approximately $10^{7}$ ions over the plasma membrane surface solves for its resting potential, results in $10^{-20} \mathrm{~J}$ when the distance between any two potassium ions, about $10 \mathrm{~nm}$, is considered. Effectively the energy $\left(1.9 \cdot 10^{-20} \mathrm{~J}\right)$ axon's action potential, that can be inferred by $1.6 \cdot 10^{-19} \mathrm{~A} \cdot \mathrm{s}$ multiplied by the net change in voltage $\left(\Delta \mathrm{V}=1.2 \cdot 10^{-1} \mathrm{~V}\right)$, is a conservation of that energy transformed from statics to dynamics.

The $10^{-20} \mathrm{~J}$ order of magnitude as a discrete amount of energy is associated singular shifts in the bond angle of receptor proteins that allows sequestering of the ligands. The hinge motion associated with sequestering the agonist for glutamate binding is associated with $1.5 \cdot 10^{-20} \mathrm{~J}$. The energy difference between phosphorylated and unphosphorylated subunits of phenylanine hydroxylase was in the order of $1.8 \cdot 10^{-20} \mathrm{~J}$.

When we measured photon emissions from melanoma cells directly by sensitive photomultiplier units the estimated unit of energy was $10^{-20} \mathrm{~J}$ per cell [11]. We found that the most parsimonious process that would have produced this "quantity" would have been narrow phase modulations in the range of the width of a plasma cell membrane, that is about 10 to 20 $\mathrm{nm}$, for photons within the visible wavelength particularly near the ultraviolet boundary for the visible wavelengths.

These observations are congruent with the analyses of the RRM frequencies reported here for the ERK-MAP pathway. Although the peak frequencies that could be associated with this specific pathway occurred within the visible, near-ultraviolet, range, the difference in energies between these peaks were in the order of $10^{-20} \mathrm{~J}$. We suggest that this increment of energy either transports or is the unit of energy, which, when presented as temporal patterns, defines the biological functions of the pathway in a manner analogous to Cosic's concept that a specific frequency for a single molecule describes its biological function.

From an astronomical perspective, this indicates that the unit energies that either influence or determine the "information" which defines the boundaries of the cell's structure and function are the same or similar to those that might define the structures and functions of all matter. The relevance of astronomical concepts and principles in cell biology, particularly when addressing the ubiquitous and recalcitrant manifestations such as cancer and malignancy, could be much more important than imagined. Persinger and Lafrenie [12], applying the innovative and integrative work of Michael Levin [13], have shown quantitative evidence that "cancer" cells may reflect a more universal phenomena coupled to sources of 
variance related to Cosmic Microwave Background (CMB) energies and to the quantities by which bits of information dissipate into or appear from entropy.

The convergence may be more apparent than anticipated. According to Cosic [2] the conductive electron transfer produced by the difference in free electron potentials at the $\mathrm{N}$ and $\mathrm{C}$ terminals of a protein can be expressed as a pseudopotential that is $0.128 \mathrm{Ry}$ or $2.78 \cdot 10^{-19}$ $\mathrm{J}$ or $1.74 \mathrm{~V}$. The maximum velocity from this energy difference would then be:

$$
\mathrm{v}_{\max }=\sqrt{ }\left(2 \mathrm{q} \cdot \mathrm{V} \cdot \mathrm{m}^{-1}\right)
$$

where $\mathrm{q}$ is the unit charge, $\mathrm{V}$ is the potential difference estimated from the pseudopotential, and $\mathrm{m}$ is the mass of the electron. The solution is $7.87 \cdot 10^{5} \mathrm{~m} \cdot \mathrm{s}^{-1}$.

For a packet of energy, such as might be contained within an electron, to move across a quintessential cell with a diameter of $\sim 10 \mu \mathrm{m}$, approximately $1.27 \cdot 10^{-11} \mathrm{~s}$ would be required. The equivalent frequency is $0.78 \cdot 10^{11} \mathrm{~Hz}$. The energy associated with that frequency, obtained by multiplying by Planck's constant $\left(6.626 \cdot 10^{-34} \mathrm{~J} \cdot \mathrm{s}\right)$ is $5.21 \cdot 10^{-23} \mathrm{~J}$. The temperature equivalent of this value from the Boltzmann constant of $1.38 \cdot 10^{-23} \mathrm{~J} \cdot \mathrm{T}^{-1}$ where $\mathrm{T}$ is ${ }^{\circ} \mathrm{K}$, would be $3.7^{\circ} \mathrm{K}$ which is within the range of Cosmic Microwave Background energies. One possible interpretation for this convergence is that the upper limit of Cosic velocity is the interface for access to or from entropy-related energies within the $\mathrm{CMB}$ as predicted by Persinger and Lafrenie [12].

\section{ELECTROMAGNETIC TRANSFORMS AND EXPERIMENTAL VERIFICATION}

Murugan et al [14] found that spring water (containing near physiological patterned ion concentrations) exposed for several days in darkness while being exposed to physiologicallypatterned (frequency and phase-modulated) magnetic fields within the microTesla range displayed conspicuous photon emissions The peak wavelengths of those emissions suggested energy associated with that exposure had been "represented" or "stored" within the organization of water. Each of the serial point durations of the voltages that comprised the magnetic temporal patterns were $3 \mathrm{~ms}$. This value had been selected because of the empirical demonstration of its efficacy for producing powerful biological effects on both organisms and cells. The value had been derived from the nearest integer solution from the predictions of Persinger and Koren [15] for the time required for a proton to expand one Planck's Length according to cosmological concepts derived from the Hubble parameter. There is experimental evidence for this solution [16].

When $1 \mathrm{cc}$ cuvettes of this exposed water was measured for fluorescence intensity between 320 and $470 \mathrm{~nm}$, there was an increase of about 150 photon counts per unit wavelength within the 420 to $440 \mathrm{~nm}$ range. The peak of the shift $(399 \mathrm{~nm})$ between the activated magnetic fields (4.4 to $11.5 \mu \mathrm{T})$ and the weaker $(409 \mathrm{~nm})$ magnetic fields $(0.1$ to $0.6 \mu \mathrm{T})$ was about $10 \mathrm{~nm}$. In other words it was the spatial shift $(\lambda)$ of the classic plasma cell membrane. That narrow increments of energy were essential for the effect was indicated by the measurement for the background exposed water whose peak was $381 \mathrm{~nm}$. The relationship might be considered to be non-linear because the higher intensity effects were more similar to the background (ambient) power frequencies fields that are encountered within the laboratory environment.

Spectral analysis of the photon emissions from the water that had been exposed to the optimal intensity magnetic fields in the dark for several days before the photon emissions 
were measured revealed peaks in SPD at functional distances of $10 \mathrm{~nm}$ and $5 \mathrm{~nm}$. The shift in wavelength between the photons emissions from the water that had been exposed to the optimal magnetic field intensities and reference group would have been equivalent to about $10^{-20} \mathrm{~J}$. This is the same order of magnitude as the energy quantity associated with the differences between the peak wavelengths that describe the ERK-MAP pathway according to the Cosic' solutions.

These marked similarities which may reflect congruence reiterates that water, often described as "the solvent of Life", at pH levels compatible with living systems may be more than a passive medium. Instead it may contain the "blueprint" or directive structure for the serial activity that traditionally defines signaling pathways. From this context the serial sequence of proteins within a pathway or more appropriately network, such as ERK-MAP, would be the aggregate form of the proton-to-proton displacements in the hydronium ion that have been described by Grotthuss-type mechanisms.

Application of the same patterned magnetic field with the identical point durations (3 ms) has between demonstrated by experiment to produce incremental shifts (toward alkalinity) in $\mathrm{pH}$ in spring water during several hours of exposure. Fractional temporal increments of observation indicated that the shifts occurred for about 20 to $25 \mathrm{~ms}$. Although perhaps spurious it may be relevant that the time required to add a nucleotide to a DNA sequence or during the process of transcription has been estimated to be within this range. Thus the same configuration of magnetic field that produced the shift in the wavelength of the emission of photons can also produce transient shifts in energy that could potentially affect the dynamics of the addition of a nucleotide into a DNA process.

This converge of temporal parameters and energies between the specific features of magnetic fields that affects shifts within $\mathrm{pH}$ in spring water only, photon emissions, and the Cosic solutions for the ERK-MAP pathway indicates that the "oscillations of the physical field" that propagate through the water dipoles could be electromagnetic in nature as she predicted. In addition the capacity for this oscillation through these electromagnetic fields is contained within the ionic relationships within the water itself. Appropriately configured and applied magnetic fields access these physical capacities such that energy can be stored within this process and later be released as photons within the visible or near-visible waveband. The wavelengths are shifted or phase-modulated by values that facilitate the occurrence of $10^{-20} \mathrm{~J}$ of energetic quantities.

There could be two physical manifestations that interface between traditional matterbased translation of information between the surface of the cell and the nucleus. The first would occur through the more well known structural changes within causal series of molecules. The second could be measured as resonance electromagnetic patterns (such as photons) that are mediated through the ubiquitous but ephemeral proton of the hydronium ion. Its properties and densities should be reflected quantitatively.

Diffusion velocity of a proton according to the diffusivity term obtained by dividing the proton magnetic moment by the unit charge $\left(0.88 \cdot 10^{-7} \mathrm{~m}^{2} \cdot \mathrm{s}^{-1}\right)$ for a classic $10 \mu \mathrm{m}$ (whose energy equivalence is $10^{-20} \mathrm{~J}$ ) width cells with a surface area of $3.14 \cdot 10^{-10} \mathrm{~m}^{2}$ would require about 3 to $3.5 \mathrm{~ms}$ to traverse the volume. If the volume occupied by the nucleus is considered the time would approach $3 \mathrm{~ms}$, which is the optimal increment of time for the point durations of the applied magnetic fields to produce the diminishment effects upon cell growth. Point durations of 1, 2, 4, or $5 \mathrm{~ms}$ are much less effective [17].

There should be convergence of quantification between well known features of classical physics and the temporal progress of the information from the resonance components across the molecular sequences for the MAP-ERK pathway. This feature should differentiate the 
identification of the most likely molecular species and mass concentration that might constitute this "physical" substrate. We considered one of the most likely analogues of candidates for this identification to be drift velocity which is defined as:

$$
\mathrm{v}=\mathrm{I}(\mathrm{nAq})^{-1}
$$

where $\mathrm{v}$ is the drift velocity of the carrier particle, $\mathrm{I}$ is the net current being mediated, $\mathrm{n}$ is the number of particles in a mole based upon molar density, $\mathrm{A}$ is the area through which the current is mediated, and $\mathrm{q}$ is the unit charge.

If one assumes the direct of the information carried as quantities of energy manifested as particles is from the membrane to the nucleus and that energy utilized from glucose-related metabolism per cell is about $10^{-12} \mathrm{~J}$ per s, then an estimated number of discrete reactions could be obtained. If the basic energetic unit of these interactions is $10^{-20} \mathrm{~J}[10]$, then there would be $10^{8}$ unit reactions per second [4] with an associated current of $1.6 \cdot 10^{-19} \mathrm{~A} \cdot \mathrm{s}$ or $1.6 \cdot 10^{-11} \mathrm{~A}$ per second. When converted to $\mathrm{cm}^{-2}$ this would be about $\sim 0.5 \mu \mathrm{A} \cdot \mathrm{cm}^{-2}$ which is within range of some empirical measurements for cell currents.

If the mediator is the proton from the hydronium ion, then at a typical $\mathrm{pH}$ of 7.4 , the concentration of $\mathrm{H}^{+}$would be $3.98 \cdot 10^{-8} \mathrm{M}$ such that the functional density would be $\left[\left(1 \mathrm{~g} \cdot \mathrm{cc}^{-}\right.\right.$ $\left.\left.{ }^{1}\right) /\left(18 \mathrm{~g} \cdot \mathrm{Mol}^{-1}\right)\right] \cdot\left(3.98 \cdot 10^{-8} \mathrm{Mol}\right.$ for $\left.\mathrm{H}^{+}\right) \cdot\left(10^{6} \mathrm{cc} \cdot \mathrm{m}^{-3}\right) \cdot\left(6.023 \cdot 10^{23}\right.$ molecules $\left.\cdot \mathrm{Mol}^{-1}\right)$, or $1.33 \cdot 10^{21}$ molecules of $\mathrm{H}^{+}$per cubic $\mathrm{m}$. The product of this value with the surface area $(\mathrm{A})$ of the cell $\left(3.14 \cdot 10^{-10} \mathrm{~m}^{2}\right)$ and the unit charge of a proton $\left(1.6 \cdot 10^{-19} \mathrm{~A} \cdot \mathrm{s}\right)$ results in a denominator that when divided into the intrinsic current (I) results in a value of $2.4 \cdot 10^{-4} \mathrm{~m} \cdot \mathrm{s}^{-1}$ as a model drift velocity.

The time required for this "drift" to occur across 0.5 the width of the cell soma width to impinge upon the nucleus would be about $20 \mathrm{~ms}$. The increment is within the order of magnitude of the time required to add a nucleotide to a DNA sequence. Although the precision of this timing would clearly be related to the intrinsic current moving across the cell and its surface area, the role of $\mathrm{pH}$ becomes particularly important from this perspective. A shift of only 0.5 of a $\mathrm{pH}$ unit around $\mathrm{pH} 7.4$ could be sufficient to affect the drift velocity to values that might precisely overlap with the optimal duration for adding or preventing the addition of nucleotides to a replicating DNA sequence.

\section{THE EMERGENCE OF EXCESS CORRELATION AND ENTANGLEMENT}

The occurrence of photon emissions (or absorptions) in molecular pathways as predicted by the results of the present analyses of Cosic's RRM introduces the possibility that under specific conditions excess correlations could occur at nontraditional distances between the same pathways in different cells. The cells could be separated at great distance within an organism or potentially at great distance between organisms. The extent of this non-locality remains to be experimentally determined.

However we [18] have shown that two photoluminescent reactions separated by $10 \mathrm{~m}$ but sharing the same changing, angular velocity electromagnetic fields behaved as if the loci had been transiently superimposed. The two separate loci displayed the properties of the "same space". In this condition injection of a single amount of reactant in each of two loci simultaneously resulted in the widening of the duration of the photon emission as if twice the amount had been injected into the same reaction. 
A similar effect non-locality was noted for injections of small quantities of protons (a weak acid) into spring water [19]. Continuous, simultaneous measurement of shifts in $\mathrm{pH}$ in containers separated by $10 \mathrm{~m}$ indicated that if the solutions both shared the same, specifically configured magnetic fields with changing angular velocities where the group and phase velocities were not equal, the expected increase in acidity in the injected volume was associated with a net increase in alkalinity in the other volume.

That "excess correlation" can occur with aggregates of cells when they share these similar, rotating magnetic fields, has been demonstrated by Dotta et al [20]. Recently we found that the injections of small amounts of hydrogen peroxide into plates of cancer (mouse melanoma) cells (resulting in partial mortality) exposed to similar rotating magnetic fields was associated with a comparable mortality of these cells split from the same source if they were exposed to a similar magnetic field at the time. In this instance the distance separating the two plates of cells in each experiment was about $3 \mathrm{~km}$. The results emerged over several days of culturing during which time each pairs of plates were exposed to the specific rotating magnetic field.

The role of signaling pathways for these cells was suggested by the requirement for some proportion of the cell population in the local stimulation (the ones that received the hydrogen peroxide) to remain alive. If there was total mortality in the local cells from the injection of the peroxide there was no change in growth in the non-local cells even if they shared the same magnetic field parameters. For the non-local cells to display the "excess correlation" or "entanglement" at least $30 \%$ of the cells in the local population had to survive the hydrogen peroxide treatment.

Cells themselves might generate their own rotating, magnetic fields. Dotta et al [21] calculated that the lateral diffusion of proteins within the plasma cell membrane had the capacity to interact with magnetic fields to generate photons. A specific intensity, around 1 $\mu$ Tesla, was predicted to generate the greatest photon emissions. As predicted this intensity elicited the largest radiant flux density when measured by photomultiplier units. Conditions that synchronize the "membrane magnetic moment" of populations of cells could potentially increase their capacity to display quantitative degrees of excess correlation that could affect the signaling pathways of cells and hence their rate of proliferation or diminishment.

\section{CONCLUSIONS}

The physical-chemical bases of one of the most well known signaling pathways in living cells can be described by a resonance pattern based upon de-localized electron potentials.

The congruence between the spectral density pattern of the terminal protein (cFOS) in this pathway and the weight average of the spectral density patterns of precursor patterns indicate that wave-functions with electromagnetic characteristics manifested by specific wavelengths of photon emissions may be the energetic bases to serial molecular causality.

The quantitative similarity between the energies associated with differences between the peak photon wavelengths predicted by the Cosic Model and those that exist throughout the universe even when Planck's Length is considered supports their essential function. Multiple molecular pathways that have persisted for billions of years and have been considered to be "conserved" may be present simply because of their prominence and availability rather than their criticality. 
Quantitative solutions of the drift velocities and diffusivities involving the protons within the hydronium ion of water indicate that water itself may be "progenitor" from which molecular pathways superimpose their properties.

\section{References}

[1] I. Cosic, IEEE Transactions on Biomedical Engineering 41 (1994) 1101-1114.

[2] B. T Dotta, N. J. Murugan, L. M. Karbowski, R. M. Lafrenie, M. A. Persinger, Naturwissenschaften 101 (2014) 87-94.

[3] A. D. Aczel, Entanglement: the Greatest Mystery in Physics Raincoast Books, Vancouver, 2002.

[4] B. Albert, A. Johnson, J. Lewis, M. Raff, K. Roberts, P. Walter, Molecular Biology of the Cell Garland Science, N.Y., 2002.

[5] I. Cosic, K. Lazar, D. Cosic, IEEE Transaction on NanoBioscience (2014)

DOI: 10.1109/TNB.2014.2365851.

[6] M. A. Persinger, S. A. Koren, International Letters of Chemistry, Physics and Astronomy 15 (2014) 80-86.

[7] T. E. Decoursey, Physiological Reivews 83 (2002) 475-579.

[8] M. A. Persinger, S. A. Koren, G. F. Lafreniere, NeuroQuantology 6 (2008) 262-271.

[9] M. A. Persinger, International Letters of Chemistry, Physics and Astronomy 12 (2014) $1-10$.

[10] M. A. Persinger, Current Medicinal Chemistry 17 (2010) 3094-3098.

[11] B. T. Dotta, C. A. Buckner, D. Cameron, R. M. Lafrenie, M. A. Persinger, General Physiology and Biophysics 30 (2011) 301-309.

[12] M. A. Persinger, R. F. Lafrenie, International Letters of Chemistry, Physics and Astronomy 17 (2014) 67-77.

[13] M. Levin, BioEssays 34 (2012) 205-217.

[14] N. J. Murugan, L. M. Karbowski, R. M. Lafrenie, M. A. Persinger, Journal of Biophysical Chemistry, in press.

[15] M. A. Persinger, S. A. Koren, International Journal of Neuroscience 117 (2007) 157-175.

[16] S. A. Koren, B. T. Dotta, M. A. Persinger, The Open Astronomy Journal 7 (2014) 1-6.

[17] C. Buckner, Effects of Electromagnetic Fields on Biological Processes are Spatial and Temporal Dependent, Ph.D. Biomolecular Sciences, Laurentian University, Sudbury, 2011.

[18] B. T. Dotta, M. A. Persinger, Journal of Biophysical Chemistry 3 (2012) 72-80.

[19] B. T. Dotta, N. J. Murugan, L. M. Karbowski, M. A. Persinger, International Journal of Physical Sciences 8 (2013) 1783-1787. 
[20] B. T. Dotta, C. A. Buckner, R. M. Lafrenie, M. A. Persinger, Brain Research 388 (2011) 77-88.

[21] B. T. Dotta, R. M. Lafrenie, L. M. Karbowski, M. A. Persinger, General Physiology and Biophysics 33 (2014) 63-73.

( Received 21 December 2014; accepted 30 December 2014) 